\title{
On neighborhoods of functions associated with conic domains
}

\author{
Nihat Yağmur
}

\section{Abstract}

Let $k-S T[A, B], k \geq 0,-1 \leq B<A \leq 1$ be the class of normalized analytic functions defined in the open unit disk satisfying

$$
\Re\left(\frac{(B-1) \frac{z f^{\prime}(z)}{f(z)}-(A-1)}{(B+1) \frac{z f^{\prime}(z)}{f(z)}-(A+1)}\right)>k\left|\frac{(B-1) \frac{z f^{\prime}(z)}{f(z)}-(A-1)}{(B+1) \frac{z f^{\prime}(z)}{f(z)}-(A+1)}-1\right| .
$$

and let $k-U C V[A, B], k \geq 0,-1 \leq B<A \leq 1$ be the corresponding class satisfying

$$
\Re\left(\frac{(B-1) \frac{\left(z f^{\prime}(z)\right)^{\prime}}{f^{\prime}(z)}-(A-1)}{(B+1) \frac{\left(z f^{\prime}(z)\right)^{\prime}}{f^{\prime}(z)}-(A+1)}\right)>k\left|\frac{(B-1) \frac{\left(z f^{\prime}(z)\right)^{\prime}}{f^{\prime}(z)}-(A-1)}{(B+1) \frac{\left(z f^{\prime}(z)\right)^{\prime}}{f^{\prime}(z)}-(A+1)}-1\right| .
$$

For an appropriate $\delta>0$, the $\delta$ neighborhood of a function $f \in k-$ $U C V[A, B]$ is shown to consist of functions in the class $k-S T[A, B]$.

\section{Introduction}

Let $\mathcal{A}$ denote the family of functions $f$ of the form

$$
f(z)=z+\sum_{n=2}^{\infty} a_{n} z^{n}
$$

Key Words: Analytic function, $k$-starlike functions, $k$-uniformly convex functions. 2010 Mathematics Subject Classification: 30C45.

Received: 8 May, 2014.

Accepted: 30 June, 2014. 
which are analytic in the open unit disk $\mathcal{U}=\{z:|z|<1\}$. The classes $S^{*}$ and $C$ are the well-known classes of starlike and convex univalent functions respectively; for details, see[4].

Noor and Malik introduced and studied the class $k-U C V[A, B]$ and the corresponding class $k-S T[A, B]$ in [10] as following:

A function $f(z) \in \mathcal{A}$ is said to be in the class $k-S T[A, B], k \geq 0,-1 \leq$ $B<A \leq 1$, if and only if,

$$
\Re\left(\frac{(B-1) \frac{z f^{\prime}(z)}{f(z)}-(A-1)}{(B+1) \frac{z f^{\prime}(z)}{f(z)}-(A+1)}\right)>k\left|\frac{(B-1) \frac{z f^{\prime}(z)}{f(z)}-(A-1)}{(B+1) \frac{z f^{\prime}(z)}{f(z)}-(A+1)}-1\right| .
$$

A function $f(z) \in \mathcal{A}$ is said to be in the class $k-U C V[A, B], k \geq 0,-1 \leq$ $B<A \leq 1$, if and only if,

$$
\Re\left(\frac{(B-1) \frac{\left(z f^{\prime}(z)\right)^{\prime}}{f^{\prime}(z)}-(A-1)}{(B+1) \frac{\left(z f^{\prime}(z)\right)^{\prime}}{f^{\prime}(z)}-(A+1)}\right)>k\left|\frac{(B-1) \frac{\left(z f^{\prime}(z)\right)^{\prime}}{f^{\prime}(z)}-(A-1)}{(B+1) \frac{\left(z f^{\prime}(z)\right)^{\prime}}{f^{\prime}(z)}-(A+1)}-1\right|
$$

It can be easily seen that

$$
f(z) \in k-U C V[A, B] \Longleftrightarrow z f^{\prime}(z) \in k-S T[A, B] .
$$

Special cases.

i. $k-S T[1,-1]=k-S T, k-U C V[1,-1]=k-U C V$, the well-known classes of $k$-starlike and $k$-uniformly convex functions respectively, introduced by Kanas and Wisniowska $[6,7]$.

ii. $k-S T[1-2 \alpha,-1]=S D(k, \alpha), k-U C V[1-2 \alpha,-1]=K D(k, \alpha)$, the classes introduced by Shams et al. in [17].

iii. $0-S T[A, B]=S^{*}[A, B], 0-U C V[A, B]=C[A, B]$, the well-known classes of Janowski starlike and Janowski convex functions respectively, introduced by Janowski [5].

Geometrically, if a function $f(z) \in k-S T[A, B]$ then $\frac{(B-1) \frac{z f^{\prime}(z)}{f(z)}-(A-1)}{(B+1) \frac{z f^{\prime}(z)}{f(z)}-(A+1)}=$ $w$ takes all values from the domain $\Omega_{k}, k \geq 0$, as

$$
\Omega_{k}=\{w: \Re w>k|w-1|\}
$$

or equivalently

$$
\Omega_{k}=\left\{u+i v: u>k \sqrt{(u-1)^{2}+v^{2}}\right\} .
$$


The domain $\Omega_{k}$ represents the right half plane for $k=0$, a hyperbola for $0<k<1$, a parabola for $k=1$ and an ellipse for $k>1$, for more details see $[10]$.

Given $\delta \geq 0$, Ruscheweyh [16] defined the $\delta$ - neighborhood $N_{\delta}(f)$ of a function $f(z) \in \mathcal{A}$ by

$$
N_{\delta}(f)=\left\{g(z): g(z)=z+\sum_{n=2}^{\infty} b_{n} z^{n}, \text { and } \sum_{n=2}^{\infty} n\left|a_{n}-b_{n}\right| \leq \delta\right\} .
$$

Ruscheweyh [16] proved among other results that $N_{1 / 4}(f) \subset S^{*}$ for $f \in C$. Sheil-Small and Silvia [18] introduced more general notions of neighborhood of an analytic function. These included noncoefficient neighbourhoods as well. Problems related to the neighborhoods and some other properties of analytic functions were considered by many authors, for example, see $[1,2,3,8,9,11$, $12,13,14]$.

In this paper, the neighborhood $N_{\delta}(f)$ for functions $f(z) \in k-U C V[A, B]$ is investigated. It is shown that all functions $g \in N_{\delta}(f)$ are in the class $k-S T[A, B]$ for certain $\delta>0$.

\section{Main Results}

In order to obtain the main results, a characterization of the class $k-S T[A, B]$ in terms of the functions in another class $k-S T^{\prime}[A, B]$ is needed. For $z \in \mathcal{U}$, a function $H_{t}$ is said to be in the class $k-S T^{\prime}[A, B]$ if the function $H_{t}$ is of the form

$$
\begin{aligned}
H_{t}(z)= & \frac{1}{(B-A)\left(1-k t \pm \sqrt{t^{2}-(k t-1)^{2}} i\right)} \\
& \times\left[\frac{z\left[B-1-(B+1)\left(k t \pm \sqrt{t^{2}-(k t-1)^{2}} i\right)\right]}{(1-z)^{2}}\right. \\
& \left.-\frac{z\left[A-1-(A+1)\left(k t \pm \sqrt{t^{2}-(k t-1)^{2}} i\right)\right]}{1-z}\right]
\end{aligned}
$$

where

$$
\left\{\begin{array}{lr}
t \geq \frac{1}{k+1}, & \text { if } 0 \leq k \leq 1 \\
\frac{1}{k+1} \leq t \leq \frac{1}{k-1}, & \text { if } k>1
\end{array}\right.
$$


Recall that for any two functions $f(z)$ and $g(z)$ given by

$$
f(z)=z+\sum_{n=2}^{\infty} a_{n} z^{n}, \quad g(z)=z+\sum_{n=2}^{\infty} b_{n} z^{n}
$$

the Hadamard product (or convolution) of $f$ and $g$ is defined by

$$
(f * g)(z)=z+\sum_{n=2}^{\infty} a_{n} b_{n} z^{n}=(g * f)(z)
$$

Lemma 2.1. A function $f$ is in the class $k-S T[A, B]$ if and only if

$$
\frac{1}{z}\left(f * H_{t}\right)(z) \neq 0, \quad z \in \mathcal{U}
$$

for all $H_{t} \in k-S T^{\prime}[A, B]$.

Proof. Let $f \in k-S T[A, B]$. Then the image of $\mathcal{U}$ under $w=u+i v=\frac{(B-1) \frac{z f^{\prime}(z)}{f(z)}-(A-1)}{(B+1) \frac{z f^{\prime}(z)}{f(z)}-(A+1)}$ lies in the conic type regions $\Omega_{k}=\left\{u+i v: u>k \sqrt{(u-1)^{2}+v^{2}}\right\}$ so that for $z \in \mathcal{U}$

$$
\frac{(B-1) \frac{z f^{\prime}(z)}{f(z)}-(A-1)}{(B+1) \frac{z f^{\prime}(z)}{f(z)}-(A+1)} \neq k t \pm \sqrt{t^{2}-(k t-1)^{2}} i
$$

where

$$
\left\{\begin{array}{lr}
t \geq \frac{1}{k+1}, & \text { if } \quad 0 \leq k \leq 1 \\
\frac{1}{k+1} \leq t \leq \frac{1}{k-1}, & \text { if } k>1 .
\end{array}\right.
$$

Thus $f \in k-S T[A, B]$ if and only if

$$
\begin{aligned}
& {\left[\left(B-1-(B+1)\left(k t \pm \sqrt{t^{2}-(k t-1)^{2}} i\right)\right) z f^{\prime}(z)\right.} \\
& \left.-\left(A-1-(A+1)\left(k t \pm \sqrt{t^{2}-(k t-1)^{2}} i\right)\right) f(z)\right] \\
& /\left[z(B-A)\left(1-k t \pm \sqrt{t^{2}-(k t-1)^{2}} i\right)\right] \neq 0
\end{aligned}
$$

or equivalently

$$
\frac{1}{z}\left(f * H_{t}\right)(z) \neq 0, \quad z \in \mathcal{U}
$$

for all $H_{t} \in k-S T^{\prime}[A, B]$. 
Lemma 2.2. If

$$
H_{t}(z)=z+\sum_{n=2}^{\infty} h_{n}(t) z^{n} \in k-S T^{\prime}[A, B], \quad z \in \mathcal{U}
$$

then

$$
\left|h_{n}(t)\right|<\frac{2 n(t+1)}{|B-A| t}
$$

where $t$ is given in (13).

Proof. Writing $H_{t}(z)=z+\sum_{n=2}^{\infty} h_{n}(t) z^{n}$, and comparing coefficients of $z^{n}$ in (8) we obtain

$$
\begin{aligned}
h_{n}(t)= & {[n(B-1)-(A-1)-(n(B+1)-(A+1)) k t} \\
& \left. \pm(n(B+1)-(A+1)) \sqrt{t^{2}-(k t-1)^{2}} i\right] \\
& /\left[(B-A)\left(1-k t \pm \sqrt{t^{2}-(k t-1)^{2}} i\right)\right]
\end{aligned}
$$

Thus

$$
\begin{aligned}
\left|h_{n}(t)\right|^{2}= & {[\mid n(B-1)-(A-1)-(n(B+1)-(A+1)) k t} \\
& \left. \pm\left.(n(B+1)-(A+1)) \sqrt{t^{2}-(k t-1)^{2}} i\right|^{2}\right] \\
& /\left|(B-A)\left(1-k t \pm \sqrt{t^{2}-(k t-1)^{2}} i\right)\right|^{2} \\
= & {\left[\{n(B-1)-(A-1)-[n(B+1)-(A+1)] k t\}^{2}\right.} \\
& \left.+(n(B+1)-(A+1))^{2}\left(t^{2}-(k t-1)^{2}\right)\right] \\
& /\left[(B-A)^{2}\left((1-k t)^{2}+t^{2}-(k t-1)^{2}\right)\right] \\
= & \frac{4(n-1)[(n B-A)(k t-1)+(n-1) k t]+[n B-A+n-1]^{2} t^{2}}{(B-A)^{2} t^{2}} .
\end{aligned}
$$

If $-1 \leq B<A \leq 1, n \geq 2$ then $n B-A<n-1$, and from (13), $k t-1<t$. So

$$
\left|h_{n}(t)\right|^{2} \leq \frac{4(n-1)^{2}(t+1)^{2}}{(B-A)^{2} t^{2}}<\frac{4 n^{2}(t+1)^{2}}{(B-A)^{2} t^{2}}
$$

and

$$
\left|h_{n}(t)\right|<\frac{2 n(t+1)}{|B-A| t} .
$$


Lemma 2.3. For a function $f \in \mathcal{A}$ and $\varepsilon \in \mathbb{C}$, define the function $F_{\varepsilon}$ by

$$
F_{\varepsilon}=\frac{f(z)+\varepsilon z}{1+\varepsilon} .
$$

If for every $\varepsilon,|\varepsilon|<\delta$ we have $F_{\varepsilon} \in k-S T[A, B]$, then for every $H_{t} \in$ $k-S T^{\prime}[A, B]$

$$
\left|\frac{1}{z}\left(f * H_{t}\right)(z)\right| \geq \delta, \quad z \in \mathcal{U} .
$$

Proof. If $F_{\varepsilon} \in k-S T[A, B]$ for every $\varepsilon,|\varepsilon|<\delta$, then by Lemma 2.1 for all $H_{t} \in k-S T^{\prime}[A, B]$

$$
\frac{1}{z}\left(F_{\varepsilon} * H_{t}\right)(z) \neq 0, \quad z \in \mathcal{U}
$$

or equivalently

$$
\frac{\left(f * H_{t}\right)(z)+\varepsilon z}{(1+\varepsilon) z} \neq 0 .
$$

Since $|\varepsilon|<\delta$, it is easily follows that

$$
\left|\frac{1}{z}\left(f * H_{t}\right)(z)\right| \geq \delta .
$$

Theorem 2.1. Let $f \in \mathcal{A}, \varepsilon \in \mathbb{C}$ and $\delta>0$. If for every $\varepsilon,|\varepsilon|<\delta$, we have $F_{\varepsilon} \in k-S T[A, B]$ then

$$
N_{\delta^{\prime}}(f) \subset k-S T[A, B]
$$

for

$$
\delta^{\prime}=\frac{|B-A| t}{2(t+1)} \delta
$$

where $-1 \leq B<A \leq 1$ and $\left\{\begin{array}{l}t \geq \frac{1}{k+1}, \quad \text { if } \quad 0 \leq k \leq 1 \\ \frac{1}{k+1} \leq t \leq \frac{1}{k-1},\end{array} \quad\right.$ if $k>1$.

Proof. Let $g(z)=z+\sum_{n=2}^{\infty} b_{n} z^{n} \in N_{\delta^{\prime}}(f)$. For any $H_{t} \in k-S T^{\prime}[A, B]$,

$$
\begin{aligned}
\left|\frac{1}{z}\left(g * H_{t}\right)(z)\right| & =\left|\frac{1}{z}\left(f * H_{t}\right)(z)+\frac{1}{z}\left((g-f) * H_{t}\right)(z)\right| \\
& \geq\left|\frac{1}{z}\left(f * H_{t}\right)(z)\right|-\left|\frac{1}{z}\left((g-f) * H_{t}\right)(z)\right|
\end{aligned}
$$


From lemma 2.3,

$$
\begin{aligned}
\left|\frac{1}{z}\left(g * H_{t}\right)(z)\right| & \geq \delta-\left|\sum_{n=2}^{\infty} \frac{\left(b_{n}-a_{n}\right) h_{n}(t) z^{n}}{z}\right| \\
& \geq \delta-\sum_{n=2}^{\infty}\left|\left(b_{n}-a_{n}\right)\right|\left|h_{n}(t)\right| \\
& \geq \delta-\frac{2(t+1)}{|B-A| t} \sum_{n=2}^{\infty} n\left|\left(b_{n}-a_{n}\right)\right| .
\end{aligned}
$$

Using Lemma 2.2 and noting that $g \in N_{\delta^{\prime}}(f)$ and whence $\sum_{n=2}^{\infty} n\left|\left(b_{n}-a_{n}\right)\right|<\delta^{\prime}$, thus

$$
\left|\frac{1}{z}\left(g * H_{t}\right)(z)\right| \geq \delta-\frac{2(t+1)}{|B-A| t} \delta^{\prime}=0
$$

Therefore $\left|\frac{1}{z}\left(g * H_{t}\right)(z)\right| \neq 0$ in $\mathcal{U}$ for all $H_{t} \in k-S T^{\prime}[A, B]$ if

$$
\delta^{\prime}=\frac{|B-A| t}{2(t+1)} \delta
$$

By Lemma $2.1 \mathrm{~g} \in k-S T[A, B]$. This proves that $N_{\delta^{\prime}}(f) \subset k-S T[A, B]$.

Lemma 2.4. ([15])If $\phi$ is a convex univalent function with $\phi(0)=0=\phi^{\prime}(0)-$ 1 in $\mathcal{U}$ and $g$ is starlike univalent in $\mathcal{U}$, then for each analytic function $F$ in U,

$$
\frac{(\phi * F g)(z)}{(\phi * g)(z)} \subset \overline{c o} F(\mathcal{U}), \quad(z \in \mathcal{U})
$$

where $\overline{c o}$ stands for the closed convex hull.

Lemma 2.5. If $\phi(z) \in C, f(z) \in k-S T[A, B]$ and $g(z)=(B+1) z f^{\prime}(z)-$ $(A+1) f(z) \in S^{*}$ then $(\phi * f)(z) \in k-S T[A, B]$.

Proof. Assume $F(z)=\frac{(B-1) \frac{z f^{\prime}(z)}{f(z)}-(A-1)}{(B+1) \frac{z f^{\prime}(z)}{f(z)}-(A+1)}$. Then for $G(z)=(\phi * f)(z)$, we have $z G^{\prime}(z)=\phi(z) * z f^{\prime}(z)$. 
Hence

$$
\begin{aligned}
& \frac{(B-1) \frac{z G^{\prime}(z)}{G(z)}-(A-1)}{(B+1) \frac{z G^{\prime}(z)}{G(z)}-(A+1)}=\frac{(B-1) \frac{\phi(z) * z f^{\prime}(z)}{(\phi * f)(z)}-(A-1)}{(B+1) \frac{\phi(z) * z f^{\prime}(z)}{(\phi * f)(z)}-(A+1)} \\
& =\frac{\phi(z) *\left[(B-1) z f^{\prime}(z)-(A-1) f(z)\right]}{\phi(z) *\left[(B+1) z f^{\prime}(z)-(A+1) f(z)\right]} \\
& =\frac{\phi(z) *\left[\frac{(B-1) \frac{z f^{\prime}(z)}{f(z)}-(A-1)}{(B+1) \frac{z f^{\prime}(z)}{f(z)}-(A+1)}\right]\left[(B+1) z f^{\prime}(z)-(A+1) f(z)\right]}{\phi(z) *\left[(B+1) z f^{\prime}(z)-(A+1) f(z)\right]} \\
& =\frac{\phi(z) *(F g)(z)}{\phi(z) * g(z)} \text {. }
\end{aligned}
$$

By Lemma 2.4 the image of $\mathcal{U}$ under $\frac{\phi(z) *(F g)(z)}{\phi(z) * g(z)}$ is a subset of the convex hull of $F(\mathcal{U})$. That means $(\phi * f)(z) \in k-S T[A, B]$.

Theorem 2.2. If $f \in k-U C V[A, B]$ and $g(z)=(B+1) z f^{\prime}(z)-(A+1) f(z) \in$ $C$ then $F_{\varepsilon}=\frac{f(z)+\varepsilon z}{1+\varepsilon} \in k-S T[A, B]$ for $|\varepsilon|<\frac{1}{4}$.

Proof. Let $f(z)=z+\sum_{n=2}^{\infty} a_{n} z^{n} \in k-U C V[A, B]$. Then

$$
\begin{aligned}
F_{\varepsilon} & =\frac{f(z)+\varepsilon z}{1+\varepsilon} \\
& =\frac{z(1+\varepsilon)+\sum_{n=2}^{\infty} a_{n} z^{n}}{1+\varepsilon} \\
& =\frac{f(z) *\left\{z(1+\varepsilon)+\sum_{n=2}^{\infty} z^{n}\right\}}{1+\varepsilon} \\
& =f(z) * \frac{\left(z-\frac{\varepsilon}{1+\varepsilon} z^{2}\right)}{1-z}=f(z) * h(z)
\end{aligned}
$$

where $h(z)=\frac{z-\frac{\varepsilon}{1+\varepsilon} z^{2}}{1-z}$. Now,

$$
\frac{z h^{\prime}(z)}{h(z)}=\frac{1-2 \rho z+\rho z^{2}}{(1-\rho z)(1-z)}, \quad \text { where } \rho=\frac{\varepsilon}{1+\varepsilon} .
$$

Hence $|\rho|=\frac{|\varepsilon|}{1-|\varepsilon|}<\frac{1}{3}$ gives $|\varepsilon|<\frac{1}{4}$. Thus

$$
\Re\left(\frac{z h^{\prime}(z)}{h(z)}\right) \geq \frac{1-2|\rho||z|-|\rho||z|^{2}}{(1-|\rho||z|)(1+|z|)}>0 .
$$


This inequality holds for all $|\rho|<\frac{1}{3}$ which is true for $|\varepsilon|<\frac{1}{4}$. Therefore $h$ is starlike in $\mathcal{U}$ and so

$$
\int_{0}^{z} \frac{h(t)}{t} d t=z+\sum_{n=2}^{\infty} \frac{c_{n}}{n} z^{n}=h(z) * \log \left(\frac{1}{1-z}\right)
$$

is convex for $|\varepsilon|<\frac{1}{4}$

$$
(f * h)(z)=z f^{\prime}(z) *\left[h(z) * \log \left(\frac{1}{1-z}\right)\right]
$$

$f(z) \in k-U C V[A, B] \Longrightarrow z f^{\prime}(z) \in k-S T[A, B]$ and $h(z) * \log \left(\frac{1}{1-z}\right) \in C$.

Also $g(z) \in C$ implies that $z g^{\prime}(z) \in S^{*}$. By Lemma 2.5, we have

$$
z f^{\prime}(z) *\left[h(z) * \log \left(\frac{1}{1-z}\right)\right] \in k-S T[A, B]
$$

Thus

$$
(f * h)(z)=\frac{f(z)+\varepsilon z}{1+\varepsilon} \in k-S T[A, B] .
$$

Theorem 2.3. Let $f \in k-U C V[A, B]$ then

$$
N_{\delta^{\prime}}(f) \subset k-S T[A, B]
$$

for

$$
\delta^{\prime}=\frac{|B-A| t}{8(t+1)}
$$

where $-1 \leq B<A \leq 1$ and $\left\{\begin{array}{lr}t \geq \frac{1}{k+1}, \quad \text { if } \quad 0 \leq k \leq 1 \\ \frac{1}{k+1} \leq t \leq \frac{1}{k-1}, & \text { if } k>1\end{array}\right.$

Proof. Let $f \in k-U C V[A, B]$ then we get the result by taking $\delta=\frac{1}{4}$ in Theorem 2.1 and using Theorem 2.2.

\section{References}

[1] R.M. Ali, K.G. Subramanian, V. Ravichandran and O.P. Ahuja, Neighborhoods of starlike and convex functions associated with parabola, J. Ineq. and Appl., 10.1155 (2008) 346279. 
[2] O. Altıntaş and S. Owa, Neighborhoods of certain analytic functions with negative coefficients, Int. J. Math. Sci., 19 (1996) 797-800.

[3] O. Altıntaş, H. Irmak, H. M. Srivastava, Neighborhoods for certain subclasses of multivalently analytic functions defined by using a differential operator, Comput. Math. Appl. 55 (2008), no. 3, 331-338.

[4] A.W. Goodman, Univalent Functions, vols. I-II, Mariner Publishing Company, Tempa, Florida, USA, 1983.

[5] W. Janowski, Some extremal problems for certain families of analytic functions, Ann. Polon. Math. 28 (1973) 297-326.

[6] S. Kanas and A. Wisniowska, Conic regions and $k$ - uniform convexity, J. Comput. Appl. Math., 105 (1999) 327-336.

[7] S. Kanas and A. Wisniowska, Conic domains and starlike functions, Rev. Roumaine Math. Pures Appl., 45 (2000) 647-657.

[8] B. S. Keerthi, A. Gangadharan, H. M. Srivastava, Neighborhoods of certain subclasses of analytic functions of complex order with negative coefficients, Math. Comput. Modelling 47 (2008), no. 3-4, 271-277.

[9] G. Murugusundaramoorthy, H. M. Srivastava, Neighborhoods of certain classes of analytic functions of complex order, J. Inequal. Pure Appl. Math. 5 (2004), no. 2, Article 24, 8 pp. (electronic).

[10] K.I. Noor, S.N. Malik, On coefficient inequalities of functions associated with conic domains, Comput. Math. Appl. 62, (2011), no.5, 2209-2217.

[11] H. Orhan, E. Kadioğlu, Neighborhoods of a class of analytic functions with negative coefficients, Tamsui Oxf. J. Math. Sci. 20 (2004), no. 2, 135-142.

[12] H. Orhan, M. Kamali, Neighborhoods of a class of analytic functions with negative coefficients, Acta Math. Acad. Paedagog. Nyházi (N.S.) 21 (2005), no. 1, 55-61 (electronic).

[13] H. Orhan, On neighborhoods of analytic functions defined by using Hadamard product, Novi Sad J. Math. 37 (2007), no. 1, 17-25.

[14] H. Orhan, Neighborhoods of a certain class of p-valent functions with negative coefficients defined by using a differential operator, Math. Inequal. Appl. 12 (2009), no. 2, 335-349. 
[15] St. Ruscheweyh and T. Sheil-Small, Hadamard products of Schlicht functions and the Polya-Schoenberg conjecture, Comm. Math. Helv., 48 (1973) 119-135.

[16] St. Ruscheweyh, Neighborhoods of univalent functions, Proceedings of the American Mathematical Society, 81 (1981) 521-527.

[17] S. Shams, S.R. Kulkarni, J.M. Jahangiri, Classes of uniformly starlike and convex functions, Int. J. Math. \& Math. Sci. 55 (2004) 2959-2961.

[18] T. Sheil-Small and E.M. Silvia, Neighborhoods of analytic functions, Journal d'Analyse Mathematique, vol. 52, (1989) 210-240.

Nihat Yağmur,

Department of Mathematics,

Faculty of Science and Art,

Erzincan University,

24000, Erzincan, Turkey.

Email: nhtyagmur@gmail.com 
ON NEIGHBORHOODS OF FUNCTIONS ASSOCIATED WITH CONIC 